\title{
Roles of NOD1/Rip2 signal pathway in carotid artery remodelling in spontaneous hypertensive rats
}

\author{
Jingsi Zhang ${ }^{1, *}$, Bingqian Fang ${ }^{1,2, *}$, Lingmin Sun ${ }^{3}$, Xiuzhen Zhang ${ }^{3}$, Jinli Liu ${ }^{1}$, Yun Yang 4 , \\ Wenhua Zhang ${ }^{4}$, Xiuli Wang ${ }^{3}$ and Yanchun Ding ${ }^{1}$ \\ ${ }^{1}$ Department of Cardiology II, The Second Hospital of Dalian Medical University, Dalian, China \\ ${ }^{2}$ Department of Internal Medicine, Shaoxing Central Hospital, Shaoxing, China \\ ${ }^{3}$ Department of Histology and Embryology, Dalian Medical University, Dalian, China \\ ${ }^{4}$ Department of Ultrasonography, The Second Hospital of Dalian Medical University, Dalian, China
}

\begin{abstract}
Nucleotide-binding and oligomerization domain (NOD) receptor is a member of inherent immunity recognition receptor family. We investigated the NOD1/Rip2 signalling pathway on carotid arterial remodelling in spontaneously hypertensive rats (SHRs). SHRs were treated with NOD1 agonist (iE-DAP), inhibitor (ML130), or normal saline. We determined the NOD1 and Rip2 expression in carotid artery tissues, serum tumour necrosis factor- $\alpha$ (TNF- $\alpha$ ) and monocyte chemotactic protein-1 (MCP-1). The carotid artery remodelling in 16-week SHRs was higher than that of 8-week SHRs and 16-week Wistar-Kyoto (WKY) rats. Expression of NOD1, Rip2, MCP-1 and TNF- $\alpha$ in 16-week SHRs was higher than that of 8-week SHRs and 16-week WKY rats. Blood pressure in iE-DAP-treated SHRs was higher than SHR-C group (no treatment), together with MCP-1, TNF- $\alpha$, NOD1 and Rip2 expression, as well as carotid artery remodelling. In ML130-treated group, these aspects were completely the opposite. Taken together, inhibition of NOD1/Rip2 signalling pathway could delay the vascular remodelling process.
\end{abstract}

Key words: Hypertension - Carotid artery remodelling - NOD1/Rip2 signal pathway - Inflammation - Blood pressure

Abbreviations: DPB, diastolic blood pressure; NOD, nucleotide-binding and oligomerization domain; MCP-1, monocyte chemotactic protein-1; SBP, systolic blood pressure; SHRs, spontaneously hypertensive rats.

\section{Introduction}

Vascular remodelling is the most prevalent form of target organ damages in the hypertensive patients (Park and Schiffrin 2001). Arterial structure and functional reconfiguration, defined as adaptation reaction of the hemodynamic distur-

\footnotetext{
* These authors contributed equally to this work.

Correspondence to: Yanchun Ding, Department of Cardiology II, The Second Hospital of Dalian Medical University, Dalian 116023, China

E-mail: yanchunding@aliyun.com
}

bance, neurohumor alternation and local endocrine secretion (Navar 2014; Chen et al. 2015), have been considered as the major pathological basis for target organ (e.g. heart, brain and kidney) damages and the hypertensive progression. Nowadays, the exact mechanism of hypertension-induced vascular remodelling is still not well defined. On this basis, prevention and reverse of hypertension-induced vascular remodelling has been proposed for treating hypertension and related complications (Gupta and Guptha 2010). In a previous study, aberrant immunoregulation may induce persistent inflammation in vascular system and the target organs, which then triggered the vascular remodelling,

(c) The Authors 2022. This is an open access article under the terms of the Creative Commons Attribution-NonCommercial 4.0 International License (https://creativecommons.org/licenses/by-nc/4.0/), which permits non-commercial use, distribution, and reproduction in any medium, provided the original work is properly cited. 
sclerosis, endothelial injury and vasomotor dysfunction that finally led to deterioration of organ damages (Lai et al. 2017; Dai et al. 2018).

Persistent inflammatory reaction played important roles in the vascular remodelling process among hypertension patients. Nucleotide-binding and oligomerization domain (NOD) receptor has been acknowledged as an important member of inherent immunity pattern recognition receptor family (Wiese et al. 2017). NOD receptor-mediated inflammation was closely associated with the pathogenesis of multiple cardiovascular diseases (Chen et al. 2015). As a major member of NOD family, NOD1 protein-mediated signalling pathways play important roles in inflammatory regulation and involve in the pathogenesis and progression of multiple chronic inflammation including atherosclerosis (Correa et al. 2012). Upon activation of NOD1 signalling pathway, expression of downstream inflammatory factors was induced, which then mediated the proliferation of vascular smooth muscle and alternation of phenotype (Gupta and Guptha 2010), as well as activation of macrophage and phenotype alternation (Dai et al. 2018). The binding of NOD1 and its receptor led to aggregation of receptorinteracting protein 2 (Rip2), which then activated NF- $\mathrm{BB}$ and secretion of downstream inflammatory factors such as monocyte chemotactic protein-1 (MCP-1) and tumour necrosis factor- $\alpha$ (TNF- $\alpha$ ) (Navar 2014). MCP-1 could trigger the activation of mononuclear macrophage and T lymphocytes, resulting in secretion of inflammatory factors and fibrogenic factors. TNF- $\alpha$ involved in regulation of several immune responses that participated in the vascular damages in the presence of endogenous chemokines, which was closely related to the pathogenesis of hypertension and vascular remodelling (Lai et al. 2017). Nevertheless, their roles in the pathogenesis of hypertension and the target organ damages are still unclear.

In this study, we determined the expression of genes and proteins involved in the NOD1/Rip2 signalling pathway in spontaneously hypertensive rats (SHRs). In addition, we investigated the effects of agonist and inhibitor of NOD1 receptor on blood pressure and carotid artery remodelling in SHRs.

\section{Material and Methods}

\section{Animals}

The animals, purchased from the Charles River Biotech (Peking, China, approval No SCXK 2012-0001), were housed in the Animal Centre of Dalian Medical University facilitated with specific pathogen-free (SPF) barrier system. The animal handling was conducted according to the Guide for the Care and Use of Laboratory Animals issued by US National Institutes of Health (No. 85-23, 1996). Experiments were performed under a project license (NO.: SCXK 20120001) granted by the Ethical Committee of Dalian Medical University.

The experiments were performed after adaptive feeding at the SPF Centre for 1 week. The protocols were divided into two parts. In part I, we compared blood pressure, carotid artery remodelling, NOD1 and Rip2, TNF- $\alpha$ and MCP-1 expression in Wistar-Kyoto (WKY) rats and SHRs at age 8 weeks $(n=5), 12$ weeks $(n=5)$ and 16 weeks $(n=5)$. In part II, the SHRs ( $n=20,8$-weeks-old) were randomly divided into four groups: SHR-H group, SHRs subjected to administration of $\mathrm{iE-DAP}$ (the NOD1 receptor specific agonist, Invivogen; $350 \mu \mathrm{g} / 100 \mathrm{~g}$ ); SHR-I group, SHRs subjected to administration of ML130 (NOD1 receptor specific inhibitor, Abcam; $100 \mu \mathrm{g} / 100 \mathrm{~g}$ ); SHR-NS group, SHRs subject to equal volume of normal saline, and SHR-C group, SHRs received no treatment. The drugs were given after 1-week feeding in these SHRs. The agents (iE-DAP or ML130) or the normal saline were given via peritoneal injection every other day for 2 weeks ( 7 times in total). After feeding for 1 week, the blood sample and carotid artery tissues were obtained.

\section{Determination of blood pressure}

The blood pressure was determined using the non-invasive facility (BP-300A, Kangtai Biotech, Chengdu, China) under a conscious state to the caudal artery. The rats were fixed in the cages, with the abdominal wall downwards. The ambient temperature was at $32^{\circ} \mathrm{C}$. The systolic blood pressure (SBP) and diastolic blood pressure (DBP) were measured for at least 5 times, in order to obtain the average.

\section{Analysis of carotid arterial remodelling}

The animals were anesthetized using $10 \%$ chloral hydrate, followed by blood sample collection from abdominal aorta. Upon washing with precooled phosphate buffer saline (PBS), the left carotid artery was fixed using $10 \%$ formalin. The sections $(5 \mu \mathrm{m})$ were subject to hematoxylin and eosin staining, and then were observed under the inverted phase contrast microscope. Ten fields were randomly selected, and three sections were selected for each vessel in order to calculate the media thickness. The structure inside the elastic membrane was categorized into intima according to the histological features, while that between the internal and external elastic membrane was the media membrane. NISElements software was used for the measurement of internal and external diameter of carotid artery, media thickness, ratio of media thickness to lumen diameter, as well as the cross-section area of media. 


\section{Real-time PCR}

Total RNA was extracted from the right common carotid artery tissues (TransGen Biotech, Peking, China). The RNA was initially reversely transcripted into cDNA using the $5 \times$ PrimeScript commercial kit. Specific primers synthesized by Invitrogen (CA, USA) were listed in Table 1 . The mRNA level was normalized by beta-actin. PCR reaction was performed in a total volume of $10 \mu$ containing $2 \times S Y B R$ Premix, each specific primer (200 nM), and $1 \mu \mathrm{l}$ cDNA template. The PCR conditions consisted of denaturation at $95^{\circ} \mathrm{C}$ for $5 \mathrm{~min}$, followed by 40 cycles of denaturation at $95^{\circ} \mathrm{C}$ for $30 \mathrm{~s}$, annealing at $54^{\circ} \mathrm{C}$ for $30 \mathrm{~s}$, and extension at $72^{\circ} \mathrm{C}$ for $15 \mathrm{~s}$. Finally, the results were analyzed using the $2^{-\Delta \Delta \mathrm{CT}}$ method.

\section{Western blot analysis}

The tissues obtained from right carotid artery were homogenized in RIPA lysis buffer containing protease and phosphatase inhibitors. Proteins were separated by electrophoresis on a $10 \%$ SDS-PAGE gel and transferred to a Hybond-P PVDF membrane. The membrane was blocked in 5\% nonfat milk and incubated with a primary antibody (1:1000, Santa Cruz, CA, USA) overnight at $4^{\circ} \mathrm{C}$. Then the mixture was incubated with the peroxidase-conjugated rabbit anti-goat secondary antibody (1:1000, Bioss, Peking, China) for $1 \mathrm{~h}$ at room temperature. After washing with PBS, the bound primary antibody was visualized with the ImageJ software and exposed to film. The same membrane probed for $\beta$-actin served as loading control.

\section{Immunohistochemical staining}

The expression of NOD1 and Rip2 was also observed by semi-quantitative analysis of 3,3'-diaminobenzidine (DAB) staining method. The NOD1 and Rip2 positive signals were stained in a brown colour, which was expressed in the cytoplasm. The staining results of the carotid artery were observed under an inverted phase contrast microscope. Ten fields were randomly selected for each slice to measure the proliferation index by calculating percentage of proliferating cell nuclear antigen positive staining cells to the total media cells.

\section{ELISA}

Blood samples $(5 \mathrm{ml}$ ) were obtained from abdominal aorta of each rat. Serum TNF- $\alpha$ and MCP-1 were determined using ELISA with commercial kits (Langdun Biotech, Shanghai, China), according to the manufacturer's instructions. Finally, the OD value was determined at a wavelength of $450 \mathrm{~nm}$.
Table 1. Primer sequences

\begin{tabular}{lll}
\hline \multirow{2}{*}{ Gene } & \multicolumn{2}{l}{ Primer sequences } \\
\hline \multirow{2}{*}{ NOD1 } & Forward & 5'-TAGCCTTCTGCAATGCTTGTTC-3' \\
& Reverse & 5'-CCGTGAGACGGCTAAAGCAA-3' \\
\cline { 2 - 3 } Rip2 & Forward & 5'-CGTCGAAGGCGTACCATTG-3' \\
& Reverse & 5'-GTGGGCGCTCTGAACTTTTC-3' \\
\cline { 2 - 3 } k-actin & Forward & 5'-CCTGTGGCATCCATGAAACTAC-3' \\
& Reverse & 5'-CCAGGGCAGTAATCTCCTTCTG-3' \\
\hline
\end{tabular}

\section{Statistical analysis}

SPSS 18.0 software was used for the data analysis. Measurement data were presented as mean \pm standard deviation. Analysis of variance (ANOVA) and repeated ANOVA with post hoc were performed for the inter-group comparison. A $p$ value of $<0.05$ was considered to be statistically significant.

\section{Results}

\section{Blood pressure in SHRs and WKY rats at different ages}

We compared the blood pressure of SHRs and WKY rats at 8,12 and 16-week-old. The blood pressure showed a significant increase in SHRs in 12 and 16-week-old compared to 8 -week-old $(p<0.05)$, especially at 16 -week-old. At 16-week-old, the blood pressure in the SHRs was significantly higher than that of the WKY rats $(p<0.05$; Fig. $1 \mathrm{~A}$ and $\mathrm{B})$.

\section{Carotid arterial remodelling of SHRs and WKY rats at different ages}

The media thickness, ratio of media thickness to lumen diameter and the cross-section area of media were used to evaluate remodelling of carotid artery. The carotid artery remodelling in 16-week-old SHRs was significantly severe than that of 8 -week-old SHRs $(p<0.05)$. In 16-week-old SHRs, these indices were significantly higher than those of 16 -week-old WKY rats $(p<0.05)$. There were no differences at week 16 in WKY rats compared with that of WKY at week 8 ( $p>0.05$; Fig. 2A and B; Table 2).

\section{NOD1 and Rip2 expression in SHRs and WKY rats at different ages}

Real-time PCR indicated significant increase in NOD1 and Rip2 mRNA expression in 12-week-SHRs and 16-week-SHRs compared with 8-week-SHRs $(p<0.05)$. In 16-week-SHRs, the NOD1 and Rip 2 mRNA expression in the carotid artery 
Table 2. Carotid artery remodelling of SHR and WKY rats at different ages

\begin{tabular}{|c|c|c|c|c|c|c|}
\hline \multirow{2}{*}{ Parameter } & \multicolumn{3}{|c|}{ WKY rats } & \multicolumn{3}{|c|}{ SHRs } \\
\hline & 8-week & 12-week & 16-week & 8-week & 12-week & 16-week \\
\hline Media thickness $(\mu \mathrm{m})$ & $40.09 \pm 1.96$ & $45.04 \pm 1.53$ & $51.63 \pm 2.13$ & $41.49 \pm 1.41$ & $56.71 \pm 1.28$ & $61.88 \pm 1.56^{\star, \#}$ \\
\hline Media thickness/lumen diameter (\%) & $6.96 \pm 0.34$ & $8.21 \pm 0.32$ & $7.95 \pm 0.28$ & $6.79 \pm 0.53$ & $9.13 \pm 0.73$ & $9.18 \pm 0.48^{\star, \#}$ \\
\hline Cross-section area of media $\left(\mathrm{mm}^{2}\right)$ & $0.077 \pm 0.01$ & $0.096 \pm 0.006$ & $0.12 \pm 0.006$ & $0.098 \pm 0.017$ & $0.124 \pm 0.008$ & $0.15 \pm 0.02^{\star, \#}$ \\
\hline
\end{tabular}

${ }^{*} p<0.05$ vs. 8-week-old SHRs; ${ }^{\#} p<0.05$ vs. 16-week-old WKY rats. SHRs, spontaneously hypertensive rats; WKY, Wistar-Kyoto.

showed significant increase compared with 16-week-WKY rats $(p<0.05)$. There were no statistical differences in NOD1 and Rip2 mRNA expression in 12-week and 16-week-old WKY rats compared with that of 8 -week-WKY rats $(p>$ 0.05; Fig. $3 \mathrm{~A}$ and $\mathrm{B}$ ).

Western blot analysis showed that the expression of NOD1 and Rip2 protein in the 12-week and 16-week SHRs groups was significantly higher than that of 8-week SHRs, respectively $(p<0.01)$. Expression of NOD1 and Rip2 protein in the carotid artery tissues in the 12-week and 16-week WKY groups showed no differences compared with that of 8 -week WKY rats $(p>0.05)$. NOD1 and Rip2 protein expression in 16-week SHRs group was significantly higher than that of 16-week WKY group ( $p<0.05$; Fig. 3C).

Immunohistochemical staining was used for the semiquantitative detection of NOD1 and Rip2 protein. Expres-
A

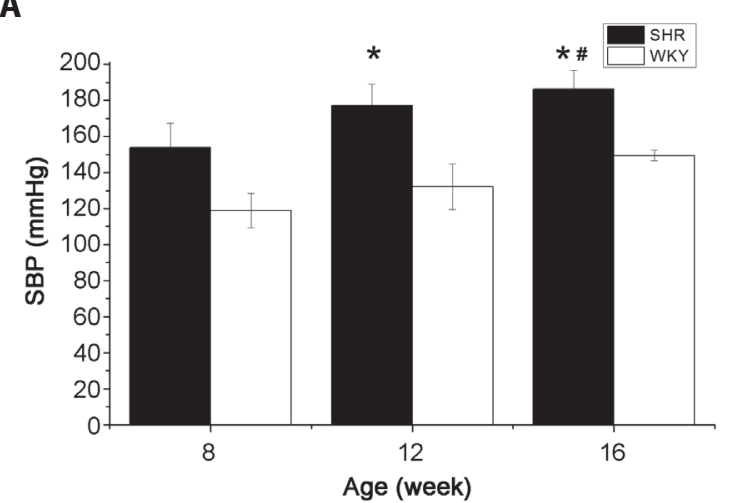

C

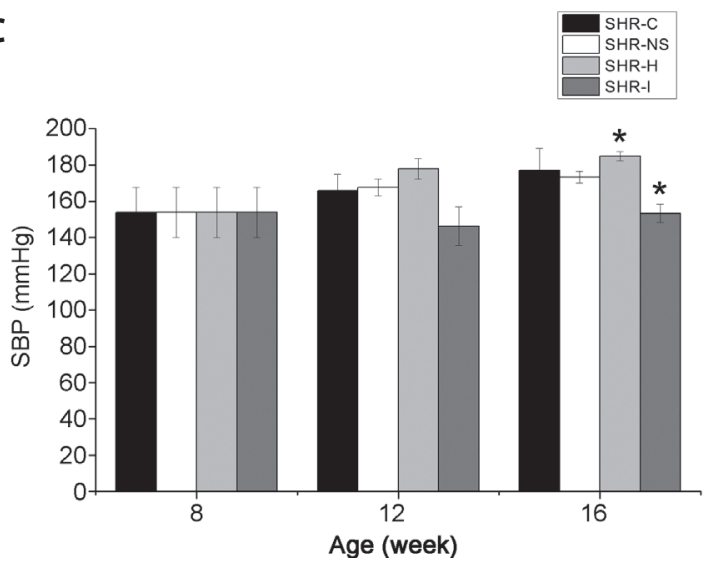

B

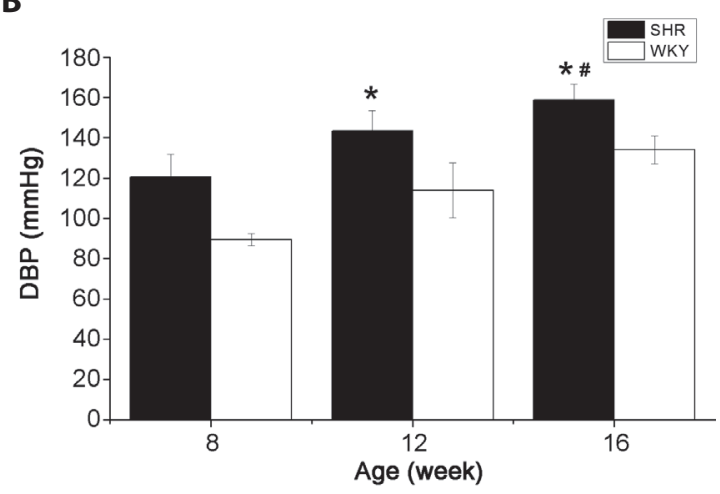

D

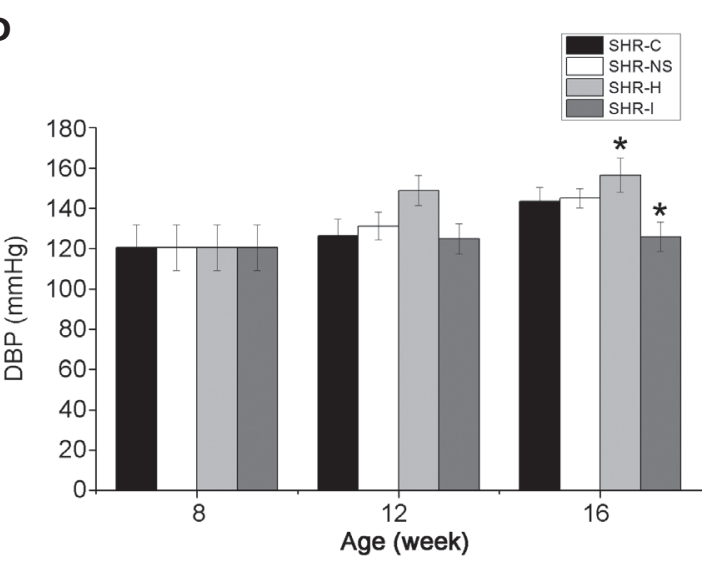

Figure 1. A., B. Comparison of blood pressure level (SBP and DBP) in SHRs and WKY rats of different ages. ${ }^{*} p<0.05 v s$. 8-week-old SHRs; ${ }^{\#} p<0.05$ vs. 16-week-old WKY rats. C., D. Effects of NOD1 agonist (iE-DAP) and inhibitor (ML130) on blood pressure level of SHRs. ${ }^{*} p<0.05$ vs. SHR-C. SBP, systolic blood pressure; DBP, diastolic blood pressure; SHRs, spontaneously hypertensive rats; SHR-H, SHRs subjected to administration of iE-DAP (350 $\mu \mathrm{g} / 100 \mathrm{~g})$; SHR-I, SHRs subjected to administration of ML130 (100 $\mu \mathrm{g} / 100 \mathrm{~g})$; SHRNS, SHRs subject to equal volume of normal saline; SHR-C, SHRs received no treatment; WKY, Wistar-Kyoto. 
sion of NOD1 and Rip2 protein in the carotid artery tissues in 12-week and 16-week SHRs group was significantly higher than that of 8-week SHRs group. There were no statistical differences in their expression in 16-week WKY group compared with that of 8 -week WKY group $(p>$ $0.05)$. The proliferation index in 16-week SHRs group was significantly higher than that of the 16-week WKY group $(p<0.05$; Fig. 4$)$.

\section{Serum MCP-1 and TNF- $\alpha$ expression in SHRs and WKY rats}

ELISA revealed that the expression of serum MCP-1 and TNF- $\alpha$ in 12-week and 16-week SHRs was significantly higher than that of 8 -week SHRs $(p<0.05)$. In contrast, there were no statistical differences in the serum TNF- $\alpha$ and MCP-1 between the 8-week, 12-week and 16-week WKY groups. Serum MCP-1 and TNF- $\alpha$ in 16-week SHRs group was significantly higher than that of 16 -week WKY group $(p<0.05$; Fig. 5A and B).

Effects of NOD1 agonist and inhibitor on the blood pressure of SHRs

With the increase of age, the blood pressure in the SHR-H, SHR-NS and SHR-C groups showed gradual elevation rather than the SHR-I group. At week 12, SBP and DBP of SHR-H group were significantly higher than that of SHR-C group $(p<0.05)$. The SBP and DBP of the SHR-I group were significantly lower than that of SHR-C group $(p<0.05)$. There were no statistical differences between SHR-NS group and SHR-C group ( $p>0.05$; Fig. $1 \mathrm{C}$ and D).

Effects of NOD1 agonist and inhibitor on the carotid artery remodelling in SHRs

At week 12, there was obvious carotid artery remodelling in the SHR-H group compared with that of the SHR-C group. There was obvious elevation in media thickness, ratio of media thickness to lumen diameter and the cross-section area of media compared with those of the SHR-C group $(p<$ 0.05). Compared with the SHR-C group, there were significant decline in media thickness, ratio of media thickness to
A

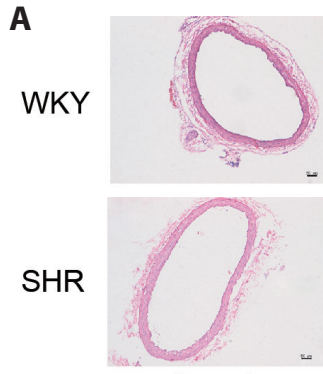

8-week

B

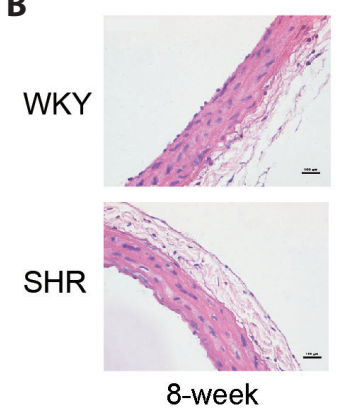

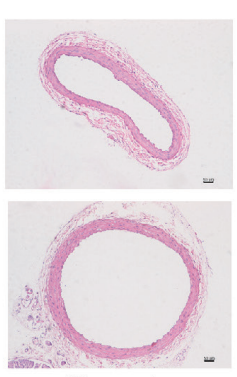

12-week

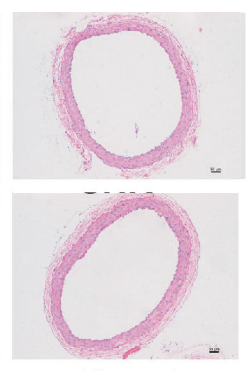

16-week

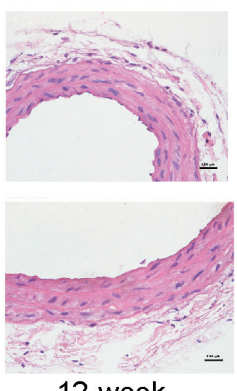

12-week

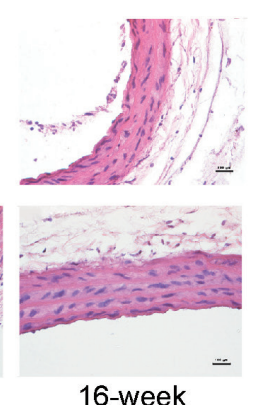

16-week
C

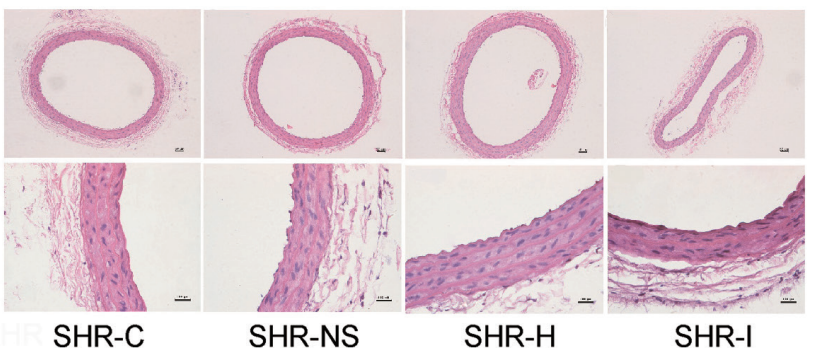

Figure 2. Images of hematoxylin and eosin staining of the carotid artery. A., B. Carotid artery from SHRs and WKY rats 8, 12 and 16-week-old at different magnification $(A \times 10, B \times 40)$. C. Carotid artery from each experimental group of SHRs at magnification: upper panel $\times 10$, bottom panel $\times 40$. For $a b-$ breviations, see Fig. 1.

lumen diameter and the cross-section area of media in the SHR-I group $(p<0.05)$. There were no statistical differences between the SHR-C group and SHR-NS group $(p>0.05$; Fig. 2C; Table 3).

Table 3. Effects of NOD1 agonist (iE-DAP) and inhibitor (ML130) on the carotid artery remodelling in SHRs

\begin{tabular}{lcccc}
\hline Parameter & SHR-C & SHR-NS & SHR-H & SHR-I \\
\hline Media thickness $(\mu \mathrm{m})$ & $56.52 \pm 1.48$ & $56.20 \pm 1.12$ & $61.88 \pm 1.56^{*}$ & $50.71 \pm 2.3^{\star}$ \\
Media thickness/lumen diameter $(\%)$ & $9.19 \pm 0.64$ & $9.18 \pm 0.73$ & $10.42 \pm 0.48^{*}$ & $8.34 \pm 0.48^{*}$ \\
Cross-section area of media $\left(\mathrm{mm}^{2}\right)$ & $0.1187 \pm 0.08$ & $0.1238 \pm 0.008$ & $0.15 \pm 0.021^{\star}$ & $0.109 \pm 0.014^{*}$ \\
\hline
\end{tabular}

${ }^{\star} p<0.05$ vs. SHR-C. NOD, nucleotide-binding and oligomerization domain; SHRs, spontaneously hypertensive rats; SHR-H, SHRs subjected to administration of iE-DAP (350 $\mu \mathrm{g} / 100 \mathrm{~g})$; SHR-I, SHRs subjected to administration of ML130 (100 $\mu \mathrm{g} / 100 \mathrm{~g})$; SHR-NS, SHRs subject to equal volume of normal saline; SHR-C, SHRs received no treatment. 
A

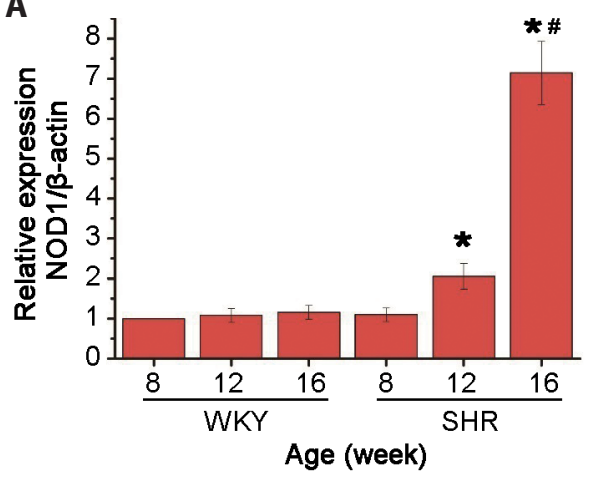

B

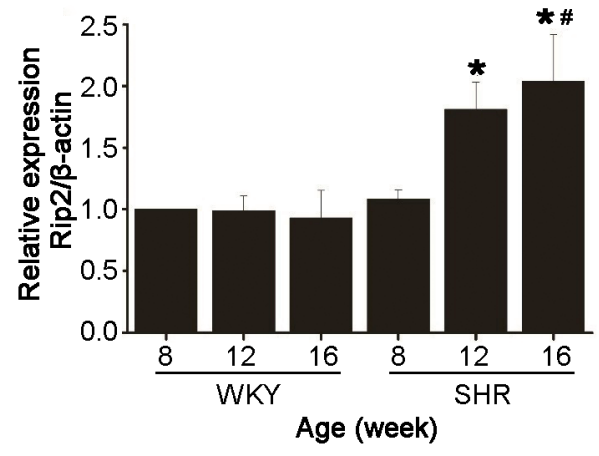

C
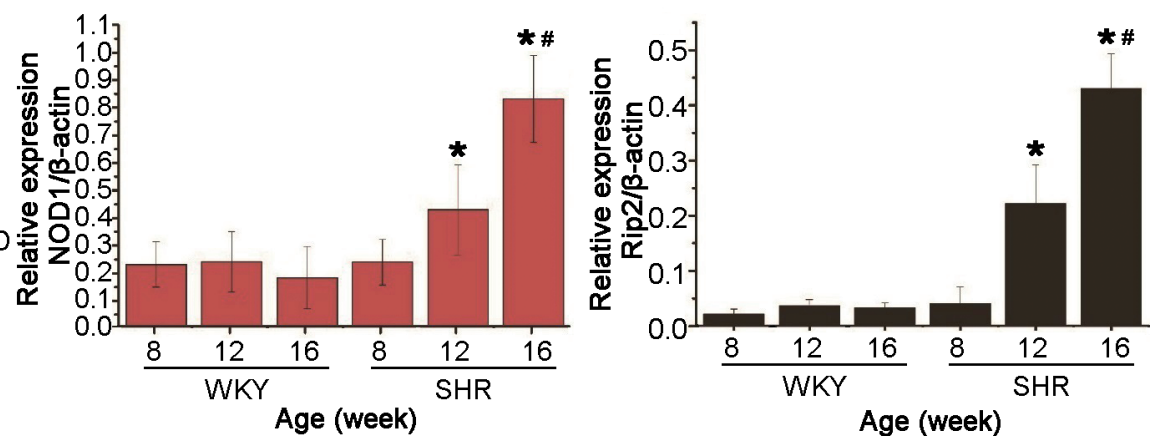

Figure 3. NOD1 and Rip2 expression of SHRs and WKY rats at different ages detected by RT PCR and Western blot. A., B. Expression of NOD1 and Rip2 mRNA. C. Protein expression of NOD1 and Rip2. ${ }^{*} p<0.05$ vs. 8-week-old SHRs; ${ }^{\#} p<0.05$ vs. 16-week-old WKY rats. For abbreviations, see Fig. 1.

Effects of NOD1 agonist and inhibitor on NOD1 and Rip2 expression

Compared with the SHR-C group, the expression of NOD1 and Rip2 mRNA in the SHR-H group showed significant elevation. The expression of NOD1 in the SHRs-H group was about 1.35-fold higher than that of the SHR-C group, while the expression of Rip2 in the SHR-H group was about 1.27 -fold higher than that of the SHR-C group $(p<0.05)$. Compared with the SHR-C group, the expression of NOD1 and Rip2 mRNA in the SHR-I group was significantly lower $(p<0.05)$. Compared with the SHR-C group, there were no statistical differences in the NOD1 and Rip2 mRNA in the SHR-NS group ( $p>0.05$; Fig. $6 \mathrm{~A}$ and B).

Compared with the SHR-C group, there was significant elevation in the expression of NOD1 and Rip2 protein in the SHR-H group. Whereas, the expression of NOD1 and Rip2 in the SHR-I group showed significant down-regulation compared with that of the SHR-C group. There were no statistical differences in the expression of NOD1 and Rip2 protein between the SHR-C group and the SHR-NS group $(p>0.05$; Fig. 6C).

Immunohistochemical staining indicated that at week 12, the proliferation index in SHR-H group was significantly higher than that of the SHR-C group $(p<0.05)$. The pro- liferation index in the SHR-I group was significantly lower than that of the SHR-C group ( $p<0.05$; Fig. 7).

\section{Expression of MCP-1 and TNF- $\alpha$ after NOD1 agonist and inhibitor}

The concentration of serum TNF- $\alpha$ and MCP-1 in the SHR$\mathrm{H}$ group was significantly higher than that of the SHR-C group $(p<0.05)$. In the SHR-I group, there was significant decline in the MCP- 1 and TNF- $\alpha$ concentration compared with that of the SHR-C group $(p<0.05)$. No statistical differences were noticed in the TNF- $\alpha$ and MCP- 1 concentration between SHR-C group and SHR-NS group $(p>0.05$; Fig. 5C and D).

\section{Discussion}

Vascular remodelling is one of the major complications for hypertensive patients. Nowadays, the exact mechanism of vascular remodelling is still not clear. In a previous study, immuno-regulatory dysfunction was considered to associate with persistent inflammation, which then led to vascular endothelial injury, activation of rennin-angiotensin system, as well as the subsequent vascular remodelling, sclerosis and 
vasomotor dysfunction (Ueda and Karas 2013). In addition, great attention has been paid to the selection of signalling pathways involved in inflammation and immune responses, in order to develop new targets for prevention and treatment of the target organ damages.

NOD receptor is an important member of the inherent immunity pattern recognition receptor family (Negroni et al. 2018). NOD receptor-mediated inflammatory responses have been revealed to be associated with the pathogenesis of cardiovascular diseases (Kharwar et al. 2016; Kim et al. 2016; Velloso et al. 2019). As the major member of NOD receptor, NOD1 could bind with the ligand to form the oligomers, which then interacted with the Caspase activation and recruitment domain (CARD) and promoted the aggregation of Rip2 (Maharana et al. 2015; Pellegrini et al. 2018). Subsequently, this would induce the aggregation of $\mathrm{NF}-\kappa \mathrm{B}$ inhibitor kinase complex, followed by degradation through the phosphorylation or polyubiquitination, together with the separation of the NF- $\kappa \mathrm{B}$ from the inhibitors (Courtois and Fauvarque 2018). Finally, its activation was triggered, which then led to the secretion of the downstream inflammatory factors (Ghosh and Febin Prabhu Dass 2016). Therefore, NOD1 receptors have been considered to play crucial roles in the pathogenesis of cardiovascular diseases and metabolic disorders (Feerick and McKernan 2017). The iE-DAP has been acknowledged as a specific agonist for NOD1 (Tan et al. 2012). The ML130 served as a potent and selective inhibitor of NOD1-dependent NF- $\kappa \mathrm{B}$ activation (Khan et al. 2011).

Heart, vessels, kidney and brain are the most common target organs of hypertension. Our previous study indicated that the expression of NOD1/Rip2 was up-regulated during the progression of myocardial remodelling in SHRs. In addition, the activation of NOD1/Rip2 signalling pathway triggered the expression of downstream cytokine IL-1, which might then lead to the aggravation of the myocardial remodelling (Liu et al. 2020). In this study, vascular remodelling was noticed in the carotid artery in the SHRs group at week 12 and 16, which was featured by increase in the media thickness of artery, ratio of media thickness to lumen
A
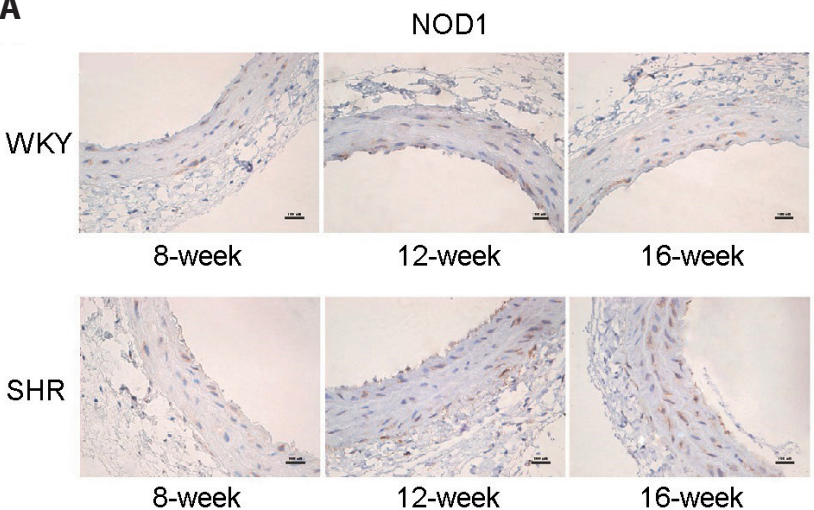

B

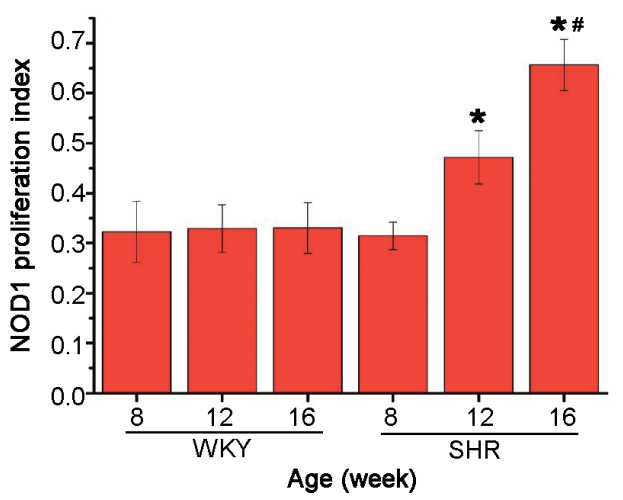

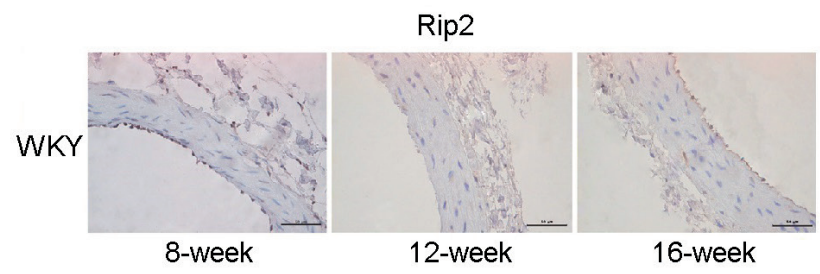

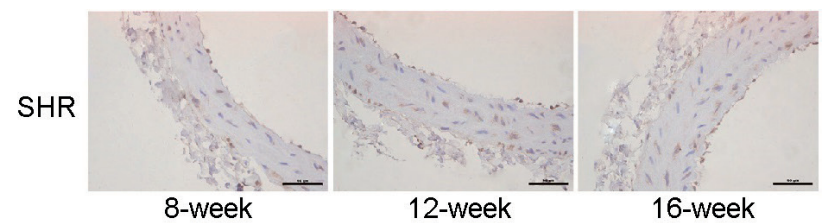

8-week

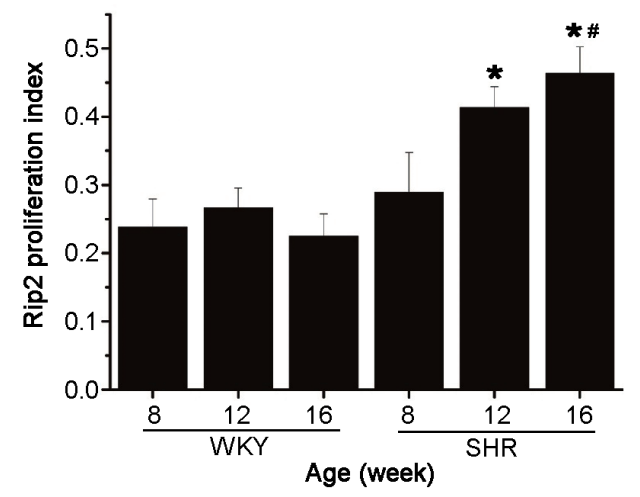

Figure 4. Semi-quantitative detection of NOD1 and Rip2 protein of SHRs and WKY rats at different ages by immunohistochemical staining. A. Immunohistochemical staining images (magnification $\times 40$ ). B. Statistical analysis of proliferation index. ${ }^{*} p<0.05 v s$. 8 -week-old SHRs; ${ }^{\#} p<0.05$ vs. 16-week-old WKY rats. For abbreviations, see Fig. 1. 

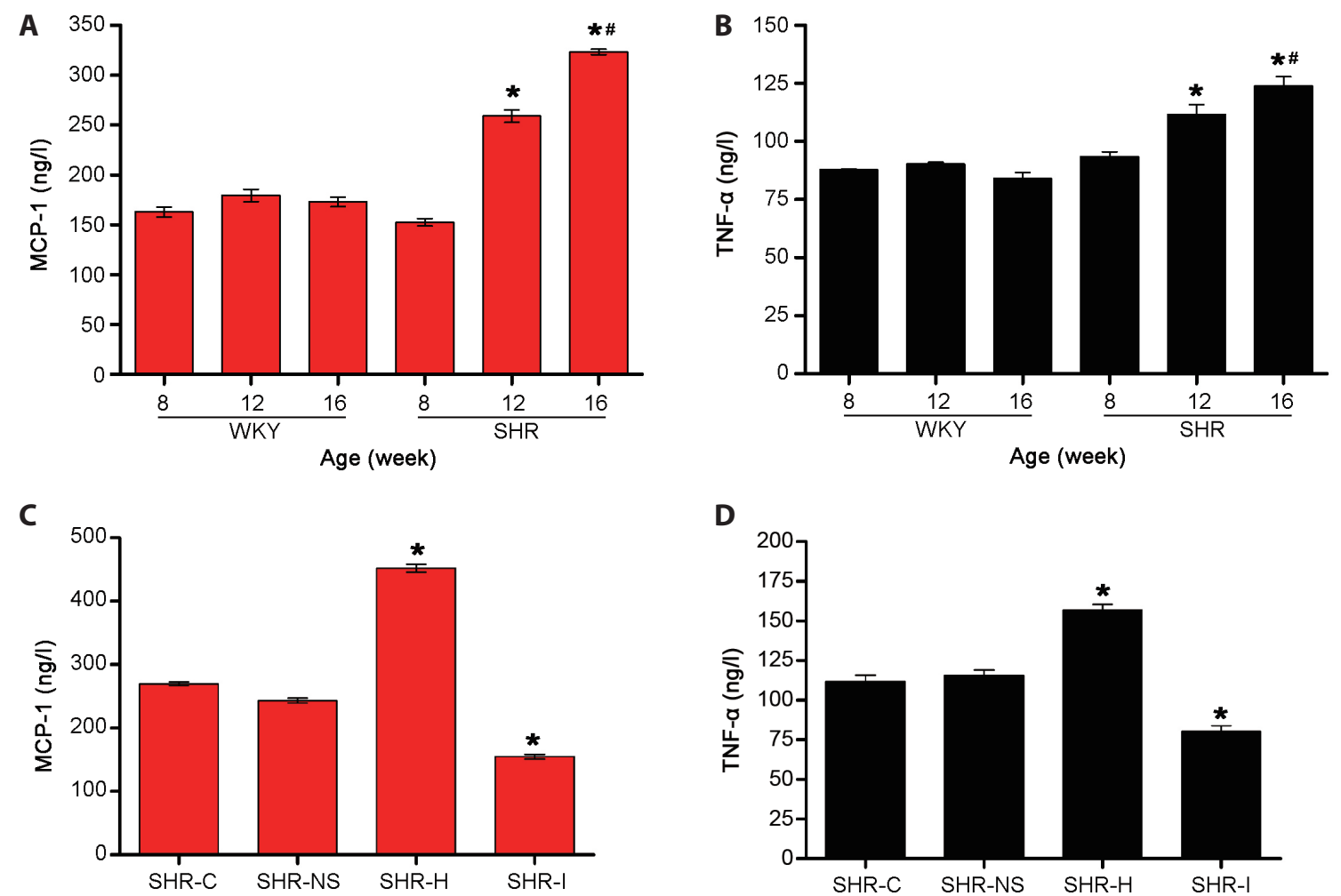

Figure 5. Serum levels of MCP-1 and TNF- $\alpha$. MCP-1 (A) and TNF- $\alpha$ (B) levels of SHRs and WKY rats at different ages. ${ }^{*} p<0.05 v s$. 8-week-old SHRs; ${ }^{*} p<0.05$ vs. 16-week-old WKY rats. MCP-1 (C) and TNF- $\alpha$ (D) levels in each experimental group of SHRs. ${ }^{*} p<$ 0.05 vs. SHR-C. For abbreviations, see Fig. 1.

diameter, as well as cross-section area of media. NOD1 and Rip2 gene and protein expression in the carotid artery of the SHRs at week 12 and 16 was significantly higher than that of SHRs at week $8(p<0.05)$. NOD1 and Rip2 gene and protein expression in the carotid artery of the SHRs at week 16 was significantly higher than that of WKY rats at week 16 $(p<0.05)$. Meanwhile, the TNF- $\alpha$ and MCP-1 expression in the downstream of NOD1/Rip2 signalling pathway was up-regulated in SHRs groups. This indicated that there might be presence of NOD1/Rip2 signalling pathway activation in the carotid artery remodelling in SHRs. In order to validate the roles of NOD1/Rip2 signalling pathway in the vascular remodelling of the carotid artery in SHRs, we then treated the SHRs with NOD1 agonist (iE-DAP) and inhibitor (ML130), respectively. We found that NOD1 agonist promoted the increase of blood pressure in SHRs, while the NOD1 inhibitor inhibited the increase of blood pressure. Our data showed that iE-DAP contributed to the pathogenesis of the carotid artery remodelling, which was featured by increase in the media thickness of carotid artery, ratio of media thickness to lumen diameter, and the cross-section area of the media. In addition, it significantly up-regulated expression of NOD1 and Rip2 gene and protein. Conversely, ML130 caused significant delay in the pathogenesis of arterial remodelling and down-regulation of NOD1 and Rip2 gene and protein in the carotid artery. These results indicated that NOD1/Rip2 signalling pathway involved in blood pressure regulation and carotid remodelling in SHRs. Vascular remodelling would trigger the increase in the peripheral vascular resistance, vasomotor dysfunction, and decline in the vascular compliance, which then contributed to the progression of hypertension (Intengan and Schiffrin 2001; Quek et al. 2016). Therefore, we considered that NOD1/Rip2 signalling pathway would affect the blood pressure through modulating the vascular remodelling process.

The activation of NOD1 signalling pathway would trigger the up-regulation of downstream inflammatory factors, and then contributed to the proliferation and phenotype changes of vascular smooth muscle cells (VSMCs) (Byndloss et al. 2016; Keestra-Gounder and Tsolis 2017; Heim et al. 2019), as well as macrophage inflammatory activation and phenotype changes (Wang et al. 2014; Mendez et al. 2019). In this study, the serum TNF- $\alpha$ and MCP-1 level increased with the progression of arterial remodelling in SHRs. The TNF- $\alpha$ and MCP-1 levels showed increase in the presence of NOD1 agonist, while they showed decrease in SHRs serum in the 
presence of NOD1 inhibitor. MCP-1 played crucial roles in the aggregation of the monocytes and macrophages in the lesion sites (Benedetti et al. 2014; Ogle et al. 2016). Upon activation of NF- $\kappa \mathrm{B}$, many cells could produce MCP-1, which could obviously up-regulate its expression (Wang et al. 2018). The subsequently activated mononuclear macrophages and $\mathrm{T}$ lymphocytes could produce inflammatory and fibrosis factors (Sanmarco et al. 2017).

TNF- $\alpha$ involves in the regulation of immune responses (Francisco et al. 2015). It could lead to vascular damages by producing endogenous chemical factors that were closely related to the hypertension and the vascular remodelling (Moghimpour Bijani et al. 2012). In a previous study, there was a negative correlation between serum TNF- $\alpha$ and arterial compliance in hypertensive patients (Verma et al. 2019). TNF- $\alpha$ could regulate the proliferation of various cells including endothelial cells, vascular smooth muscle cells (VSMCs) and myocardial cells (Choi et al. 2018). TNF- $\alpha$ could contribute to the rapid entry of VSMCs from $\mathrm{G} 1$ phase to the $S$ phase, which then promoted the VSMCs proliferation and regulated the thickness of arterial wall (Tammali et al. 2010). In addition, it could lead to secretion of IL-1 and release of vascular Angiotensin II (AngII) (Li et al. 2014). IL-1 could promote the VSMCs proliferation, which then led to thickened vascular wall, narrowed vascular lumen and increased peripheral resistance (Schultz et al. 2007). Meanwhile, AngII could lead to vasoconstriction directly. These indicated that the activation of NOD1/Rip2 in SHRs promote the expression of downstream inflammatory factors. Then it may lead to injury of endothelial cells, and aggregation of inflammatory cells in the injury sites. Moreover, it could stimulate the proliferation and migration of the VSMCs, together with the extracellular matrix changes (Kollias et al. 1999). On this basis, it could trigger the thickening of the intima and media, resulting in changes of the vascular structures and the subsequent physiologic dysfunction of the cervical vessels.

Vascular wall tension changes are major stimulating factors for the hypertension-related vascular remodelling (Renna et al. 2013). The changes of vascular tension are mainly triggered by the changes of blood pressure. Under a state of increased pressure in hypertensive patients, there was continuous elevation in the vascular wall tension, which then directly stimulated the rapid proliferation and hypertrophy of the VSMCs featured by thickening in the media (Brown et al. 2018). Moreover, the changes of blood pressure and vascular wall tension would affect the cellular apoptosis, which then involved in the vascular remodelling (Jaminon et al. 2019). In this study, both NOD1 agonist and inhibitor affected blood pressure and the progression of carotid artery remodelling.
A

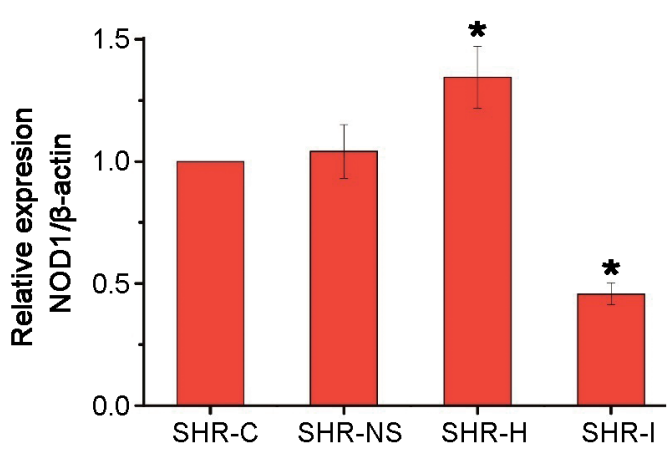

$\overline{\mathbf{B}}$

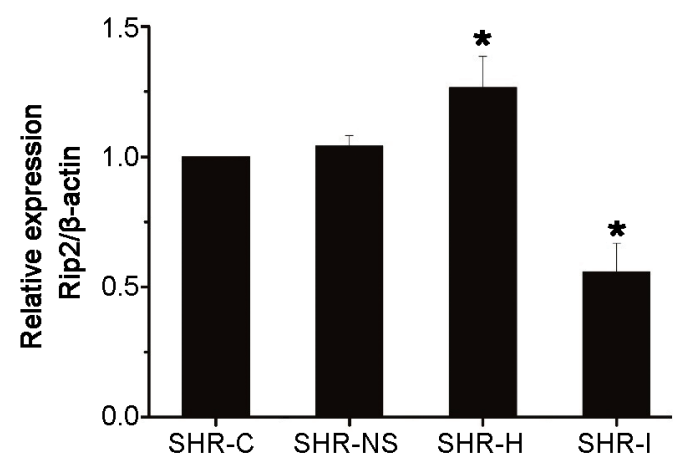

C
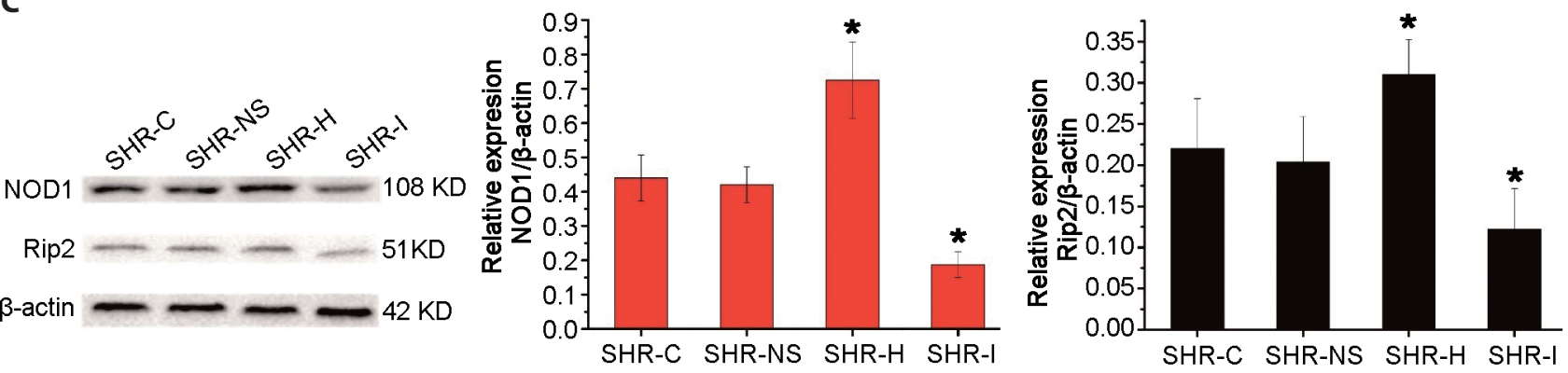

Figure 6. NOD1 and Rip2 expression in each experimental group of SHRs detected by RT PCR and Western Blot. NOD1 (A) and Rip2 (B) mRNA expression. C. NOD1 and Rip2 protein expression. ${ }^{*} p<0.05 v$ s. SHR-C. For abbreviations, see Fig. 1. 
A

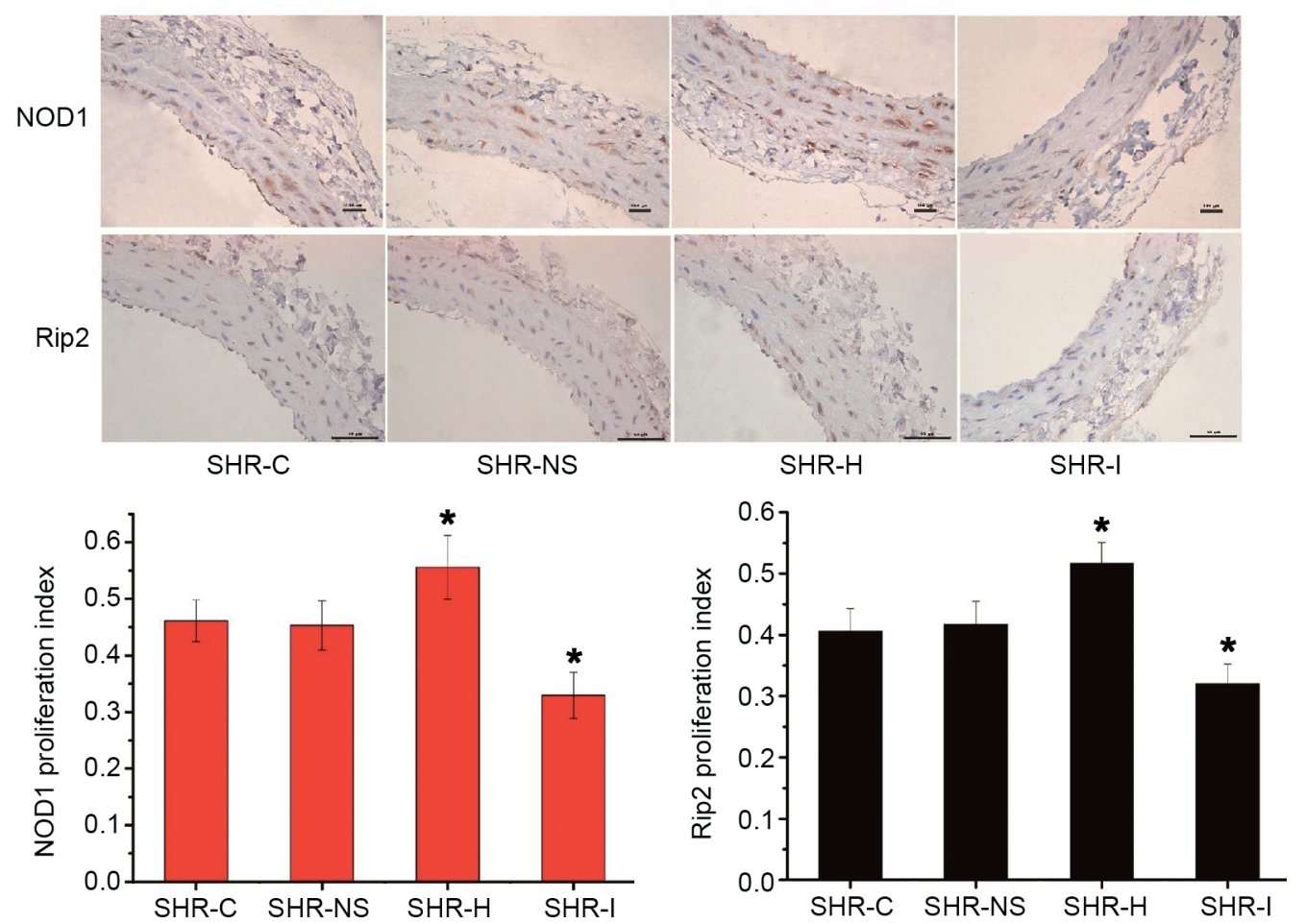

Figure 7. Semi-quantitative detection of NOD1 and Rip2 protein in each experimental group of SHRs by immunohistochemical staining. A. Immunohistochemical staining images $40 \times$. B. Statistical analysis of proliferation index. ${ }^{*} p<0.05 v s$. SHR-C. For abbreviations, see Fig. 1.

There are some limitations in our study. In this study, we only used the NOD1 agonist and inhibitor. In future, Rip2 agonist and inhibitor would be utilized in order to further verify the potential effects of such signalling pathway on the carotid artery remodelling.

In summary, our data showed that NOD1/Rip2 signalling pathway involved in the regulation of blood pressure, MCP-1 and TNF- $\alpha$ expression and carotid artery remodelling in SHRs. Inhibition of NOD1/Rip2 signalling pathway could control the blood pressure in SHRs, inhibit the expression of MCP- 1 and TNF- $\alpha$ and delay the vascular remodelling.

Conflict of interest. The authors declare that there is no conflict of interest.

Author contributions. Conception and design: JZ, YD; Provision of study materials or patients: YD; Collection and assembly of data: BF, LS, XZ; Data analysis and interpretation: JL, YY, WZ, XW; Manuscript writing: All authors; Final approval of manuscript: All authors.

Data availability. The data used to support the findings of this study are available from the corresponding author upon request.

Funding statement. No funding.

\section{References}

Benedetti F, Davinelli S, Krishnan S, Gallo RC, Scapagnini G, Zella D, Curreli S (2014): Sulfur compounds block MCP-1 production by Mycoplasma fermentans-infected macrophages through NF-kappaB inhibition. J. Transl. Med. 12, 145 https://doi.org/10.1186/1479-5876-12-145

Brown IAM, Diederich L, Good ME, DeLalio LJ, Murphy SA, Cortese-Krott MM, Hall JL, Le TH, Isakson BE (2018): Vascular smooth muscle remodeling in conductive and resistance arteries in hypertension. Arterioscler. Thromb. Vasc. Biol. 38, 1969-1985 https://doi.org/10.1161/ATVBAHA.118.311229

Byndloss MX, Keestra-Gounder AM, Baumler AJ, Tsolis RM (2016): NOD1 and NOD2: New functions linking endoplasmic reticulum stress and inflammation. DNA Cell. Biol. 35, 311-313 https://doi.org/10.1089/dna.2016.3396

Chen SL, Zhang H, Xie DJ, Zhang J, Zhou L, Rothman AM, Stone GW (2015): Hemodynamic, functional, and clinical responses to pulmonary artery denervation in patients with pulmonary arterial hypertension of different causes: phase II results from the Pulmonary Artery Denervation-1 study. Circ. Cardiovasc. Interv. 8, e002837

https://doi.org/10.1161/CIRCINTERVENTIONS.115.002837

Choi S, Park M, Kim J, Park W, Kim S, Lee DK, Hwang JY, Choe J, Won MH, Ryoo S, et al. (2018): TNF-alpha elicits phenotypic 
and functional alterations of vascular smooth muscle cells by miR-155-5p-dependent down-regulation of cGMP-dependent kinase 1. J. Biol. Chem. 293, 14812-14822 https://doi.org/10.1074/jbc.RA118.004220

Correa RG, Milutinovic S, Reed JC (2012): Roles of NOD1 (NLRC1) and NOD2 (NLRC2) in innate immunity and inflammatory diseases. Biosci. Rep. 32, 597-608 https://doi.org/10.1042/BSR20120055

Courtois G, Fauvarque MO (2018): The many roles of ubiquitin in NF-kappaB signaling. Biomedicines 6, 43 https://doi.org/10.3390/biomedicines6020043

Dai X, Hua L, Chen Y, Wang J, Li J, Wu F, Zhang Y, Su J, Wu Z, Liang C (2018): Mechanisms in hypertension and target organ damage: Is the role of the thymus key? (Review). Int. J. Mol. Med. 42, 3-12 https://doi.org/10.3892/ijmm.2018.3605

Feerick CL, McKernan DP (2017): Understanding the regulation of pattern recognition receptors in inflammatory diseases - a ,Nod in the right direction. Immunology 150, 237-247 https://doi.org/10.1111/imm.12677

Francisco NM, Hsu NJ, Keeton R, Randall P, Sebesho B, Allie N, Govender D, Quesniaux V, Ryffel B, Kellaway L, Jacobs M (2015): TNF-dependent regulation and activation of innate immune cells are essential for host protection against cerebral tuberculosis. J. Neuroinflammation 12, 125 https://doi.org/10.1186/s12974-015-0345-1

Ghosh S, Febin Prabhu Dass J (2016): Non-canonical pathway network modelling and ubiquitination site prediction through homology modelling of NF-kappaB. Gene 581, 48-56 https://doi.org/10.1016/j.gene.2016.01.025

Gupta R, Guptha S (2010): Strategies for initial management of hypertension. Indian. J. Med. Res. 132, 531-542

Heim VJ, Stafford CA, Nachbur U (2019): NOD signaling and cell death. Front. Cell. Dev. Biol. 7, 208 https://doi.org/10.3389/fcell.2019.00208

Intengan HD, Schiffrin EL (2001): Vascular remodeling in hypertension: roles of apoptosis, inflammation, and fibrosis. Hypertension 38, 581-587 https://doi.org/10.1161/hy09t1.096249

Jaminon A, Reesink K, Kroon A, Schurgers L (2019): The role of vascular smooth muscle cells in arterial remodeling: Focus on calcification-related processes. Int. J. Mol. Sci. 20, 5694 https://doi.org/10.3390/ijms20225694

Keestra-Gounder AM, Tsolis RM (2017): NOD1 and NOD2: beyond peptidoglycan sensing. Trends Immunol. 38, 758-767 https://doi.org/10.1016/j.it.2017.07.004

Khan PM, Correa RG, Divlianska DB, Peddibhotla S, Sessions EH, Magnuson G, Brown B, Suyama E, Yuan H, Mangravita-Novo A, et al. (2011): Identification of inhibitors of NOD1-induced nuclear factor- $\kappa B$ activation. ACS Med. Chem. Lett. 2, 780-785 https://doi.org/10.1021/ml200158b

Kharwar NK, Prasad KN, Paliwal VK, Modi DR (2016): Association of NOD1 and NOD2 polymorphisms with Guillain-Barre syndrome in Northern Indian population. J. Neurol. Sci. 363, 57-62 https://doi.org/10.1016/j.jns.2016.02.028

Kim YK, Shin JS, Nahm MH (2016): NOD-like receptors in infection, immunity, and diseases. Yonsei Med. J. 57, 5-14 https://doi.org/10.3349/ymj.2016.57.1.5
Kollias G, Douni E, Kassiotis G, Kontoyiannis D (1999): On the role of tumor necrosis factor and receptors in models of multiorgan failure, rheumatoid arthritis, multiple sclerosis and inflammatory bowel disease. Immunol. Rev. 169, 175-194 https://doi.org/10.1111/j.1600-065X.1999.tb01315.X

Lai CL, Xing JP, Liu XH, Qi J, Zhao JQ, Ji YR, Yang WX, Yan PJ, Luo CY, Ruan LF (2017): Relationships of inflammatory factors and risk factors with different target organ damage in essential hypertension patients. Chin. Med. J. 130, 1296-1302 https://doi.org/10.4103/0366-6999.206343

Li C, Wang Y, Qiu Q, Shi T, Wu Y, Han J, Chai X, Wang W (2014): Qishenyiqi protects ligation-induced left ventricular remodeling by attenuating inflammation and fibrosis via STAT3 and NF-kappaB signaling pathway. PLoS One 9, e104255 https://doi.org/10.1371/journal.pone.0104255

Liu FY, Fang BQ, Sun LM, Zhang XZ, Liu JL, Yang Y, Zhang WH, Wang XL, Ding YC (2020): The role of the NOD1/Rip2 signaling pathway in myocardial remodeling in spontaneously hypertensive rats. Med. Sci. Monit. 26, e924748-1-e924748-15 https://doi.org/10.12659/MSM.924748

Maharana J, Dehury B, Sahoo JR, Jena I, Bej A, Panda D, Sahoo BR, Patra MC, Pradhan SK (2015): Structural and functional insights into CARDs of zebrafish (Danio rerio) NOD1 and NOD2, and their interaction with adaptor protein RIP2. Mol. Biosyst. 11, 2324-2336 https://doi.org/10.1039/C5MB00212E

Mendez JM, Kolora LD, Lemon JS, Dupree SL, Keestra-Gounder AM (2019): Activation of the endoplasmic reticulum stress response impacts the NOD1 signaling pathway. Infect. Immun. 87, e00826-00818 https://doi.org/10.1128/IAI.00826-18

Moghimpour Bijani F, Vallejo JG, Rezaei N (2012): Toll-like receptor signaling pathways in cardiovascular diseases: challenges and opportunities. Int. Rev. Immunol. 31, 379-395 https://doi.org/10.3109/08830185.2012.706761

Navar LG (2014): Physiology: hemodynamics, endothelial function, renin-angiotensin-aldosterone system, sympathetic nervous system. J. Am. Soc. Hypertens. 8, 519-524 https://doi.org/10.1016/j.jash.2014.05.014

Negroni A, Pierdomenico M, Cucchiara S, Stronati L (2018): NOD2 and inflammation: current insights. J. Inflamm. Res. 11, 49-60 https://doi.org/10.2147/JIR.S137606

Ogle ME, Segar CE, Sridhar S, Botchwey EA (2016): Monocytes and macrophages in tissue repair: Implications for immunoregenerative biomaterial design. Exp. Biol. Med. 241, 1084-1097 https://doi.org/10.1177/1535370216650293

Park JB, Schiffrin EL (2001): Small artery remodeling is the most prevalent (earliest?) form of target organ damage in mild essential hypertension. J. Hypertens. 19, 921-930 https://doi.org/10.1097/00004872-200105000-00013

Pellegrini E, Desfosses A, Wallmann A, Schulze WM, Rehbein K, Mas P, Signor L, Gaudon S, Zenkeviciute G, Hons M, et al. (2018): RIP2 filament formation is required for NOD2 dependent NF-kappaB signalling. Nat. Commun. 9, 4043 https://doi.org/10.1038/s41467-018-06451-3

Quek KJ, Boyd R, Ameer OZ, Zangerl B, Butlin M, Murphy TV, Avolio AP, Phillips JK (2016): Progressive vascular remodelling, endothelial dysfunction and stiffness in mesenteric resistance 
arteries in a rodent model of chronic kidney disease. Vascul. Pharmacol. 81, 42-52

https://doi.org/10.1016/j.vph.2015.12.004

Renna NF, de Las Heras N, Miatello RM (2013): Pathophysiology of vascular remodeling in hypertension. Int. J. Hypertens. 2013, 808353 https://doi.org/10.1155/2013/808353

Sanmarco LM, Eberhardt N, Ponce NE, Cano RC, Bonacci G, Aoki MP (2017): New insights into the immunobiology of mononuclear phagocytic cells and their relevance to the pathogenesis of cardiovascular diseases. Front. Immunol. 8, 1921 https://doi.org/10.3389/fimmu.2017.01921

Schultz K, Murthy V, Tatro JB, Beasley D (2007): Endogenous interleukin-1 alpha promotes a proliferative and proinflammatory phenotype in human vascular smooth muscle cells. Am. J. Physiol. Heart Circ. Physiol. 292, H2927-2934 https://doi.org/10.1152/ajpheart.00700.2006

Tammali R, Saxena A, Srivastava SK, Ramana KV (2010): Aldose reductase regulates vascular smooth muscle cell proliferation by modulating G1/S phase transition of cell cycle. Endocrinology 151, 2140-2150 https://doi.org/10.1210/en.2010-0160

Tan X, Li WW, Guo J, Zhou JY (2012): Down-regulation of NOD1 in neutrophils of periparturient dairy cows. Vet. Immunol. Immunopathol. 150, 133-139 https://doi.org/10.1016/j.vetimm.2012.09.004

Ueda K, Karas RH (2013): Emerging evidence of the importance of rapid, non-nuclear estrogen receptor signaling in the cardiovascular system. Steroids 78, 589-596 https://doi.org/10.1016/j.steroids.2012.12.006

Velloso FJ, Trombetta-Lima M, Anschau V, Sogayar MC, Correa RG (2019): NOD-like receptors: major players (and targets) in the interface between innate immunity and cancer. Biosci. Rep. 39, BSR20181709 https://doi.org/10.1042/BSR20181709

Verma MK, Jaiswal A, Sharma P, Kumar P, Singh AN (2019): Oxidative stress and biomarker of TNF-alpha, MDA and FRAP in hypertension. J. Med. Life. 12, 253-259 https://doi.org/10.25122/jml-2019-0031

Wang N, Liang H, Zen K (2014): Molecular mechanisms that influence the macrophage $\mathrm{m} 1-\mathrm{m} 2$ polarization balance. Front. Immunol. 5, 614

https://doi.org/10.3389/fimmu.2014.00614

Wang Y, Ni H, Li H, Deng H, Xu LS, Xu S, Zhen Y, Shen H, Pan H, Yao M (2018): Nuclear factor kappa B regulated monocyte chemoattractant protein-1/chemokine CC motif receptor-2 expressing in spinal cord contributes to the maintenance of cancer-induced bone pain in rats. Mol. Pain. 14, 1744806918788681 https://doi.org/10.1177/1744806918788681

Wiese KM, Coates BM, Ridge KM (2017): The role of nucleotidebinding oligomerization domain-like receptors in pulmonary infection. Am. J. Respir. Cell Mol. Biol. 57, 151-161 https://doi.org/10.1165/rcmb.2016-0375TR

Received: July 27, 2021

Final version accepted: September 16, 2021 